\title{
Raman and photoluminescence characterization of focused ion beam patterned InGaN/GaN multi-quantum-wells nanopillar array
}

\author{
X. H. Wang, ${ }^{1}$ J. Q. Ning, ${ }^{2}$ S. J. Xu, ${ }^{2, a)}$ and H. W. Choi ${ }^{1, b)}$ \\ ${ }^{1}$ Department of Electrical and Electronic Engineering, The University of Hong Kong Pokfulam Road, \\ Hong Kong, China \\ ${ }^{2}$ Department of Physics and HKU-CAS Joint Laboratory on New Materials, The University of Hong Kong, \\ Pokfulam Road, Hong Kong, China
}

(Received 4 August 2011; accepted 6 October 2011; published online 10 November 2011)

\begin{abstract}
High crystal quality GaN nanopillar arrays containing InGaN/GaN multi-quantum wells (MQWs) have been fabricated by focused ion beam followed by wet etch treatments to remove the ion damage. The first order Raman spectra reveal a well-built additional peak when the diameter of the nanopillars is less than $220 \mathrm{~nm}$. This peak is also observed in the GaN pillars without MQW and is clearly assigned to the surface optical (SO) mode originating from the $\mathrm{A}_{1}$ phonon in wurtzite GaN. The frequency of this SO mode is found to be sensitive with the diameter and surface roughness of the nanopillars. Temperature-variable photoluminescence measurements show that a broadband emission in the as-grown sample split into the two well-resolved bands for nanopillars and the emission band at the higher energy side quickly thermally quenched. (c) 2011 American Institute of Physics. [doi:10.1063/1.3658866]
\end{abstract}

\section{INTRODUCTION}

With GaN emerging as a successful nitride-based semiconductor in applications of blue and ultraviolet optical devices, ${ }^{1}$ nanostructures containing InGaN/GaN multi-quantum wells (MQWs) have drawn tremendous research interests in recent years. Nanostructures can be formed either through growth (bottom-up approach) or nano-patterning followed by etching (top-down approach). Amongst established topdown approaches, focused ion beam (FIB) milling is a promising technique for the fine patterning of $\mathrm{GaN}$ nanostructures, such as dielectric-air distributed Bragg reflectors ${ }^{2}$ and nanopillar arrays. ${ }^{3}$ Most importantly, it operates in a direct-write mode which can concurrently be monitored by highresolution secondary electron imaging. The $\mathrm{Ga}^{+}$ions transfer their kinetic energy during bombardment of the crystalline sample; the process causes electron and ion emission, atomic sputtering, sample heating, and amorphization, ${ }^{4,5}$ similar to but to a greater extent compared to reactive ion plasma etching. ${ }^{6,7}$ Apart from amorphization, reports also show that $\mathrm{Ga}^{+}$ ion bombardment forms void and bubbles on the sample surface which further agglomerate into metallic nano-blitters. ${ }^{8,9}$ Hence, lateral crystal damage due to ion bombardment is inevitable. Studies on FIB damage ${ }^{4}$ show that the thickness of the amorphous layer (in the order of a few nanometers) decreases with the decrease of ion energy. However, low ion energies also result in longer patterning durations. For milling semiconductors, the milling duration for each run is normally controlled to within $10 \mathrm{~min}$ to avoid charging effects and sample heating, so that the ion beam could only be maintained at a relatively low current. Therefore, controlling the damage caused by ion bombardment is critical in

\footnotetext{
a)Electronic mail: sjxu@hku.hk.

b) Author to whom correspondence should be addressed. Electronic mail: hwchoi@hku.hk.
}

nanofabrication. While GaN epi-layers are generally chemically inert to alkaline etchants, such as $\mathrm{KOH}$ and $\mathrm{NaOH}$ aqueous solutions (with etch rates limited to tens of angstroms per minute at room temperature producing rough surfaces), ${ }^{10,11} \mathrm{KOH}$ has been shown to be an effective etchant for removal of the amorphous damaged layer. ${ }^{12}$

In this study, an array of nanopillars containing InGaN/ GaN MQW is fabricated by FIB. Raman spectroscopy is used to characterize the crystal quality of the nanopillar array before and after wet etch treatments. Through first-order Raman spectroscopy, a strong phonon peak in addition to the phonon modes allowed by symmetry is observed, attributed to the surface optical (SO) phonon. Surface phonons originate from bulk optical modes, but they are confined near the surface region of the polar crystals. ${ }^{13-15}$ The dispersion relation of the SO phonon reveals that $\omega_{\text {SO }}$ lies between the longitudinal optical (LO) and transverse optical (TO) phonons. There are three factors that affect the wave number of the SO phonon: the wave vector $k$ measured along the surface; surface geometry and the dielectric function of the surrounding medium. ${ }^{8-10}$ In most cases $\omega_{\text {SO }}$ is mainly determined by $k$, since at long wavelengths where $k \sim 0$, SO modes are hardly observable since it overlaps with the TO phonon. $k$ can be activated by breaking the translational symmetry on the lattice's surface. This can be achieved by introducing surface roughness or by construction of a grating along the surface. There have been reports of SO observation in nanostructures through Raman scattering of zinc-blend crystals such as $\mathrm{GaP},{ }^{16}$ and also in wurtzite GaN. ${ }^{17,18}$ However, most nanostructures reporting $\mathrm{SO}$ observations are fabricated by bottom-up mechanism; a systematic study on SO phonons in FIB-patterned GaN nanopillars is useful and reported in this work. Compared with bottom up methods, FIB has advantages over uniformity, dimension, and site control. In this study, the SO phonon from the FIB-patterned nanopillars is investigated from two aspects: pillar diameters and surface roughness. 


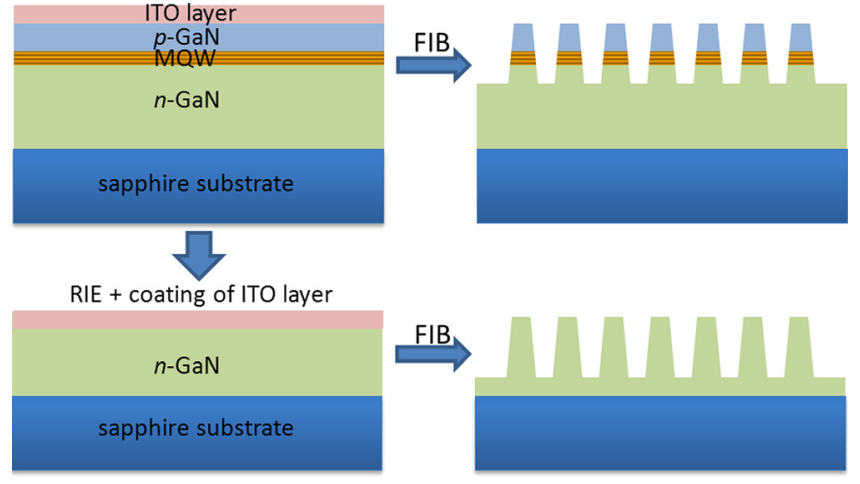

FIG. 1. (Color online) Schematic diagram illustrating FIB fabrication of nanopillars on an InGaN/GaN MQW wafer. For comparison, an identical array of nanopillars has been fabricated from a similar wafer with the MQWs etched away by RIE.

Besides confocal micro-Raman characterization, temperaturevariable photoluminescence (PL) measurements are also performed on the nanopillar array and through comparison to the as-grown wafer, significant changes in the carrier localization distribution are observed due to nanostructuring.

\section{EXPERIMENT DETAILS}

The starting wafer is an epitaxial light-emitting diode structure containing five periods of InGaN/GaN MQW grown by metal-organic chemical vapor deposition on a c-plane sapphire substrate. Figure 1 shows a schematic drawing of the wafer and fabrication process. For preparation of nanopillars, a layer of indium tin oxide (ITO) is deposited on top of the $p$-GaN to enhance surface conductivity. An excellent surface conductivity could not only prevent surface charging but also facilitate obtaining pillars of good uniformity and high-aspectratios during FIB milling. The nanopillar array is patterned with a Quanta 200 3D DualBeam (FIB/SEM) system. $30 \mathrm{keV}$ gallium ions with a beam current of $0.1 \mathrm{nA} / 0.3 \mathrm{nA}$ are used to mill the sample according to the pattern image uploaded to the software. Figure 2(a) shows the SEM image of the nanopillar array after FIB milling, with base diameters of $300 \mathrm{~nm}$. The SEM image clearly shows that an extra layer has been adhered onto the nanopillars and the sidewalls of the array boundary. This adhesive layer is attributed to the quick oxidization of the FIB damaged outer shell when the sample is exposed to air.

The samples are then subjected to successive wet etch treatments for removal of ion damage: the ITO layer and the adhesive layer are etched simultaneously in an acid solution containing $1 \mathrm{HNO}_{3}: 2 \mathrm{H}_{2} \mathrm{O}: 4 \mathrm{HCl}$. The FE-SEM image in Fig. 2(b) shows nanopillars with clean and smooth surfaces after the chemical treatment. The sample is further treated with $\mathrm{KOH}$ of $20 \mathrm{wt}$. \% dissolved in water, heated to $50{ }^{\circ} \mathrm{C}$, to remove the amorphous shell leaving the straight pillars and to reduce the base diameters of the pillars down to $180 \mathrm{~nm}$, as shown in Fig. 2(c). For comparison, another set of nanopillar arrays are fabricated by the same process, except that the $p-\mathrm{GaN}$ and MQW layers have been etched away by reactive ion etching (RIE) prior to FIB patterning, also shown in the schematic diagram of Fig. 1.

The nanopillar arrays are characterized by means of room-temperature Raman spectroscopy and temperaturevariable PL. The Raman measurements are carried out using a WITec-Alpha confocal micro-Raman system under the back scattering geometric configuration. The excitation source is an $\mathrm{Ar}+$ laser at $514.5 \mathrm{~nm}$ with an output power of $30 \mathrm{~mW}$. The laser beam is focused onto the sample with a $60 \times$ objective. For the temperature-variable PL, the excitation source is a Spectra-Physics diode-pumped solid-state (DPSS) pulsed laser emitting at $349 \mathrm{~nm}$ with a pulse width of $4 \mathrm{~ns}$ and repetition rate of $1 \mathrm{kHz}$. The sample is mounted onto the cold finger of a liquid nitrogen cooled cryostat. The PL signal is collected by an optical fiber bundle and coupled

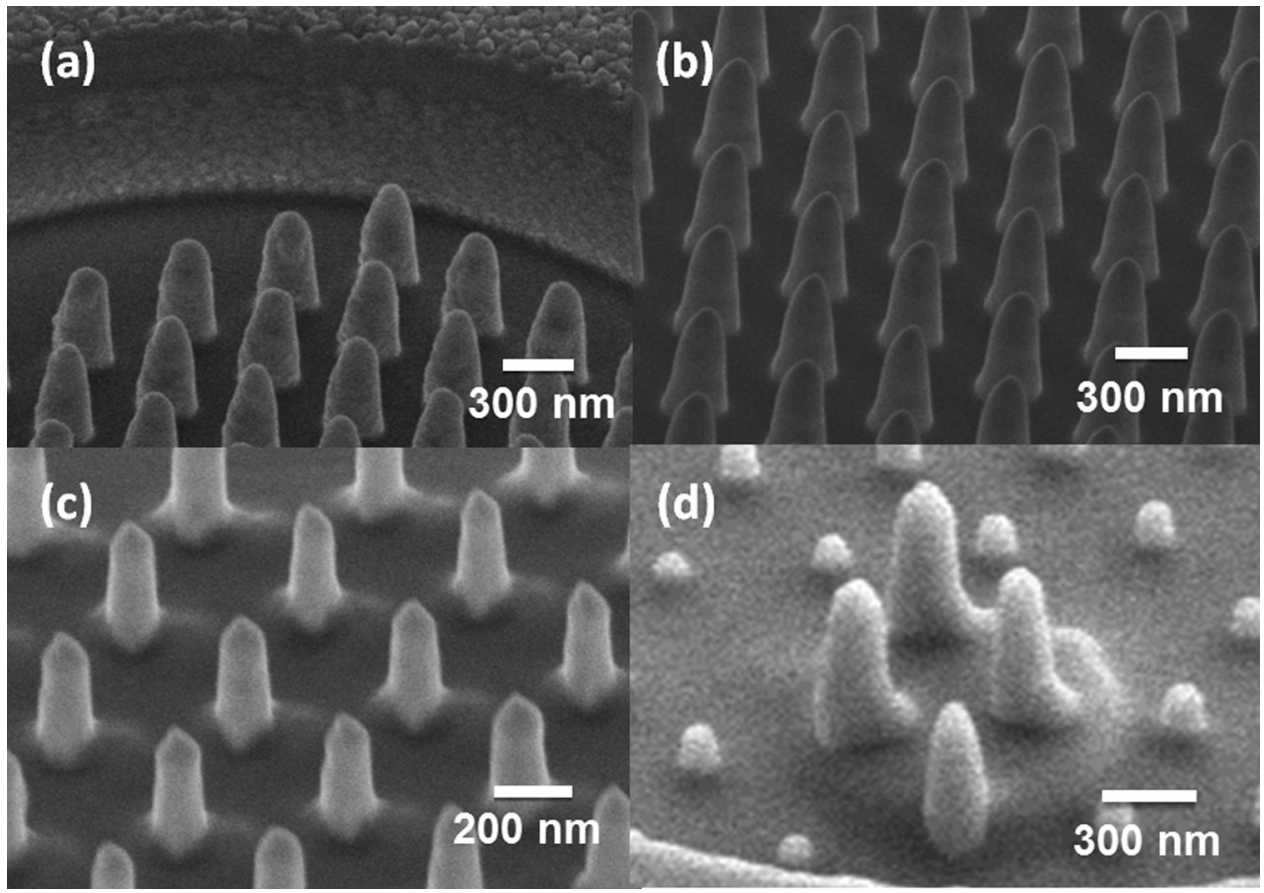

FIG. 2. SEM images showing the nanopillar array at different stages of fabrication. (a) Immediately after FIB patterning. (b) Etched in acid to remove the ITO layer as well as the amorphous GaN layer due to ion bombardment, leaving a relatively smooth surface. (c) Further etched in $\mathrm{KOH}$ solution, shrinking the diameters of the nanopillars down to approximately $150 \mathrm{~nm}$. (d) Fine milling of the array by FIB to form a small cluster of pillars. 
into an Acton SP2500A $500 \mathrm{~mm}$ spectrograph, the dispersed signal of which is detected by a Princeton Instrument PIXIS open-electrode CCD; the system offers optical resolutions of better than $0.1 \mathrm{~nm}$.

\section{RESULTS AND DISCUSSIONS}

\section{A. Raman studies on SO phonon}

Figure 3 shows the Raman spectra of the nanopillar array at different stages of wet etch treatment, and that of the as-grown wafer. For the as-grown wafer, and due to our measurement geometry of back scattering, two spectral peaks are observed at $569 \mathrm{~cm}^{-1}$ and $734 \mathrm{~cm}^{-1}$, which are unambiguously assigned to the symmetry allowed modes $\mathrm{E}_{2}$ (high) and $\mathrm{A}_{1}(\mathrm{LO})$, respectively. ${ }^{19}$ Even though the SEM image of Fig. 2(b) reveals a smooth nanopillar surface after etching in the acid solution, the Raman measurement shows broadening of the $A_{1}(L O), E_{2}($ high) phonon, and the high plateau. These are all signs of poor crystal quality on the outer shells of the nanopillars, hence indicating that the amorphous layer had not been removed by acid etching.

Three spectral peaks are observed from the Raman spectrum after $\mathrm{KOH}$ etching. The two peaks at $569 \mathrm{~cm}^{-1}$ and $736 \mathrm{~cm}^{-1}$ are again in good agreement with the phonon modes as observed in the as-grown wafer. Also the low background noise level and the sharp peaks also indicate good crystalline quality and thus suggest successful removal of the amorphous layer. The blue shift of the $\mathrm{A}_{1}(\mathrm{LO})$ peak of approximately $2 \mathrm{~cm}^{-1}$ with respect to that observed from the as-grown wafer has been explained due to the high aspect ratio of the wurtzite GaN nanopillars that truncates the dipolar sum in the nanopillar as a result of the boundary. ${ }^{13}$ In addition to the allowed modes, an additional peak at $696 \mathrm{~cm}^{-1}$ is observed, which is identified as the SO mode. In order to confirm that this SO phonon peak originates from the nanopillar structure, the array is fine-milled again by FIB, leaving behind a small cluster of 3 to 4 pillars in the center, as shown in Fig. 2(d). The additional peak at $696 \mathrm{~cm}^{-1}$ is still observed in the micro-Raman spectra from the small cluster, while the

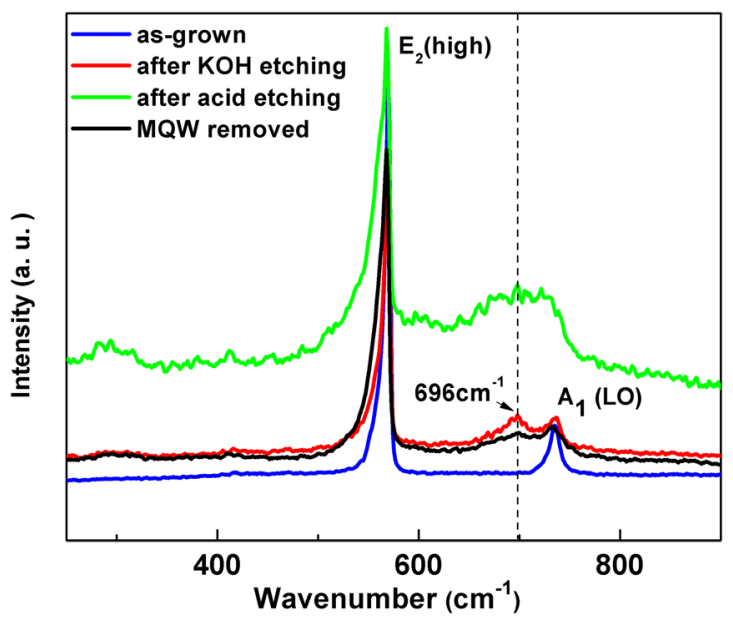

FIG. 3. (Color online) Measured Raman spectra for the as-grown wafer, MQW pillars after acid etching, MQW pillars after $\mathrm{KOH}$ etching and pillars without MQWs. spectrum for the region with pillars removed is identical to the as-grown wafer exhibiting only the bulk phonon modes. On the other hand, although the InGaN/GaN MQW region has been cited as a possible origin of this extra phonon peak ${ }^{18}$ our measured data indicates the contrary. According to the Raman spectra plotted in Fig. 3, the peak at $696 \mathrm{~cm}^{-1}$ can be observed from nanopillar arrays both with and without MQWs. Therefore, we positively attribute this new peak as the $\mathrm{SO}$ phonon associated with the nanostructure.

Theoretical studies on the dispersion relation of SO phonon using EM theory and semi-classical crystal model have well established. ${ }^{14,15}$ These studies take into account the surface modes and the finiteness of the crystal structure, whose dimensions are in the sub-micrometers range. For cylindrical interfaces ${ }^{14,16}$ the dielectric functions on both sides follow this relation,

$\varepsilon_{1}(\omega)+\varepsilon_{2}(\omega) f(k r)=0$, where $k$ is the wavevector along the surface and $r$ represents the radius of the cylinder, and

$$
f(k r)=\frac{I_{m}(k r) \mathrm{K}_{m}^{\prime}(k r)}{I_{m}^{\prime}(k r) \mathrm{K}_{m}(k r)}, \quad \mathrm{m}=0,1,2, \ldots
$$

where $I, K$ are the modified Bessel functions. Conventionally, taking the zeroth order approximation, i.e., $\mathrm{m}=0$, transformed (1) to

$$
f(k r)=\frac{I_{0}(k r) \mathrm{K}_{1}(k r)}{I_{1}(k r) \mathrm{K}_{0}(k r)},
$$

while the dielectric function for polar semiconductor is ${ }^{14}$

$$
\varepsilon_{1}(\omega)=\varepsilon_{\infty}+\frac{\varepsilon_{0}-\varepsilon_{\infty}}{1-\omega^{2} / \omega_{\mathrm{TO}}^{2}} .
$$

Rearranging the equations and taking the dielectric function of the surrounding medium to be 1 , the dispersion for $\mathrm{SO}$ phonon is given by

$$
\omega=\omega_{T O} \sqrt{\frac{\varepsilon_{0}-\varepsilon_{\infty}}{f(k r) \varepsilon_{m}+\varepsilon_{\infty}}+1} .
$$

For polar semiconductors with highly symmetric zinc-blende crystals (such as GaP and $\mathrm{ZnS}$ ), the calculation of SO phonon is relatively simple since there is only a single polar mode that splits into TO and LO modes. For anisotropic wurtzite $\mathrm{GaN}$, there exist two polar modes $\left(\mathrm{A}_{1}\right.$ and $\left.\mathrm{E}_{1}\right)$ according to the direction relative to the c-axis. SO dispersion is also affected by the measuring geometry and crystal orientation. Due to the nature of our measuring geometry which is parallel to the c-axis, only the $\mathrm{A}_{1}$ polar mode can be observed. The curve in Fig. 4 shows the calculated SO phonon dispersion of $\omega_{\text {SO }}$ versus $k r$ from the $\mathrm{A}_{1}$ phonon, having taken $\mathrm{A}_{1}(\mathrm{TO})$ as $533 \mathrm{~cm}^{-1} \varepsilon_{\infty, / /}=5.29$ and $\varepsilon_{0, / /}=10.2 .^{20}$

From the SO phonon dispersion, the wavenumber of the SO mode is dependent on the pillar geometry and its surface condition. A set of arrays with different pillar diameters are then fabricated onto the same wafer under similar FIB settings. The sample is treated by wet etching to eliminate the ion damage before Raman measurement. The base diameters for these arrays, as measured from FE-SEM images, range 


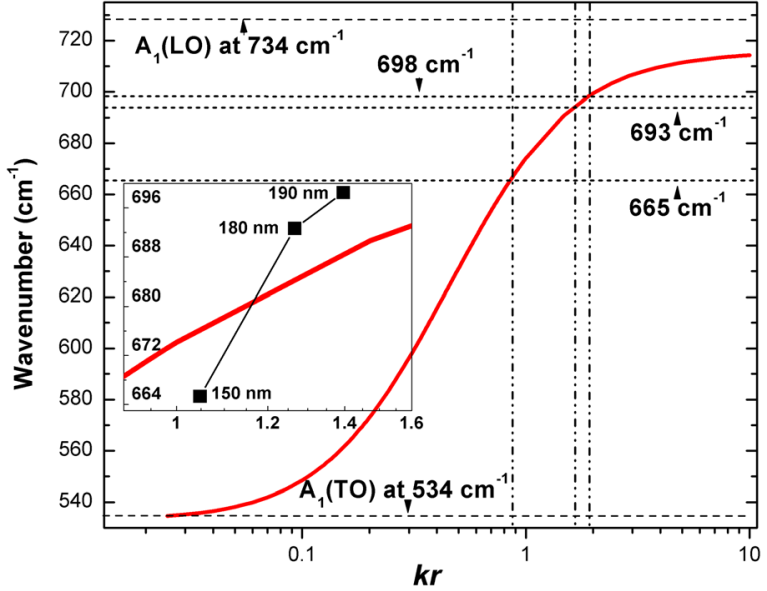

FIG. 4. (Color online) Calculated SO phonon dispersion originating from $\mathrm{A}_{1}$ phonon as a function of $k r$. The $k r$ values for experimentally observed phonon peaks at $665 \mathrm{~cm}^{-1}, 693 \mathrm{~cm}^{-1}$, and $698 \mathrm{~cm}^{-1}$ are $0.89,1.56$, and 1.85 , respectively. The inset shows the dispersion for the three pillar arrays along with the calculated curve for comparison.

from $220 \mathrm{~nm}$ to $560 \mathrm{~nm}$. As shown in Fig. 5, when the diameters of the nanopillars exceed $380 \mathrm{~nm}$, the features of the Raman spectra are identical to the as-grown background; this means that for "larger" structures, the Raman signals from the bulk GaN mode are much stronger than that from the surface region. However, for smaller nanostructures, the surface mode becomes dominant. A Raman peak at $699 \mathrm{~cm}^{-1}$ appears in the spectrum for the $220 \mathrm{~nm}$ diameter nanopillars, corresponding to the SO mode. The SO mode is further investigated using a set of nanopillar samples with diameters ranging from $190 \mathrm{~nm}$ to $150 \mathrm{~nm}$. From these obtained Raman spectra as plotted in Fig. 6, the intensities of the SO phonon peaks exceed that of the symmetry allowed $A_{1}$ phonon peak in the smaller nanopillar structures, indicating dominance of the surface modes. The $k r$ values corresponding to the various measured $\omega_{\text {SO }}$ from the nanopillars are determined from the calculated curve as represented by the vertical drop lines in Fig. 4. Since the set of samples have been processed in a similar fashion, their surface roughness and thus wavevector $k$ are expected to be identical. Based on the average value

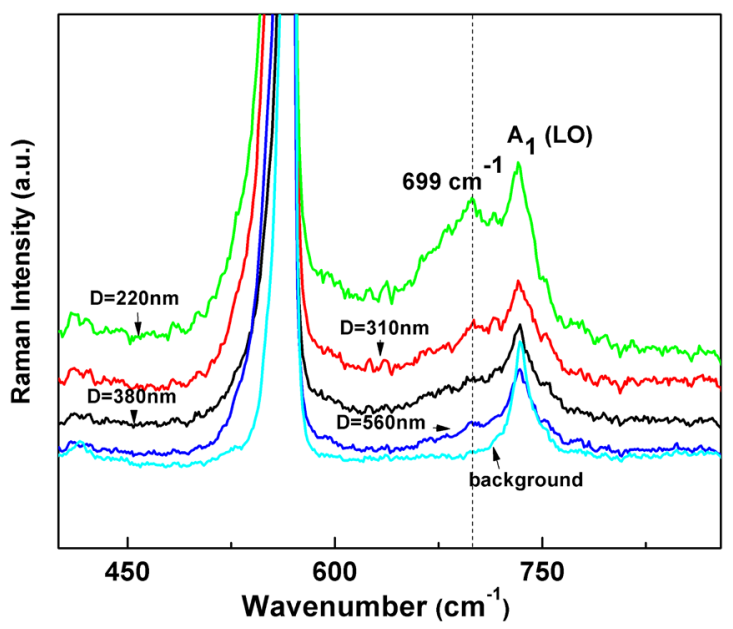

FIG. 5. (Color online) Measured Raman spectra for wet etched nanopillar arrays with different diameters, ranging from $560 \mathrm{~nm}$ to $220 \mathrm{~nm}$.

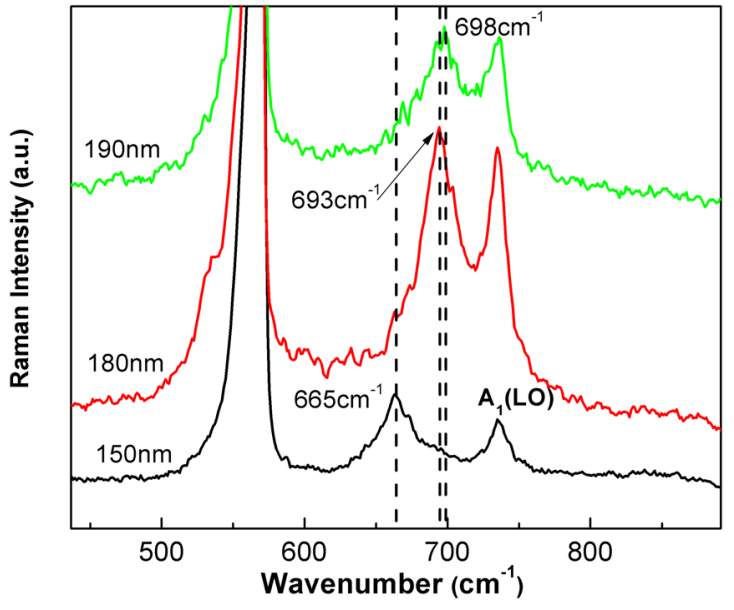

FIG. 6. (Color online) Raman spectra for nanopillar arrays with diameter ranging from $190 \mathrm{~nm}$ to $150 \mathrm{~nm}$. The spectra have been offset vertically for clarity.

of $k$ determined from $\omega_{\text {SO }}$ (evaluated as $1.4 \times 10^{7} \mathrm{~m}^{-1}$ ), the dispersion frequencies for the three pillar arrays are marked onto the plot in the inset of Fig. 4, agreeing well with the theoretical dispersion model.

To further investigate the SO phonon with regard to nanopillar surface roughness, two nanopillar arrays are prepared with identical diameters and geometry fabricated with the same FIB settings but different $\mathrm{KOH}$ treatment durations as the $\mathrm{KOH}$ treatment time is deliberately controlled to obtain different surface roughness. The pillars are examined by the low-voltage field-emission SEM and the obtained images are shown in Fig. 7. It is well known that low-energy electrons cannot penetrate deeply into the structure, thereby producing a much better surface profile. The SEM images show an obvious distinction in surface roughness between the two samples. Figure 8 shows the Raman spectra of the two arrays; the SO phonon corresponding to the smoother surface (i.e., as shown in Fig. 7(a)) has a smaller wavenumber than that of the rougher surface, and this shift of the SO phonon peak is consistent with our calculation: the smoother surface brought in a smaller disturbance to the surface
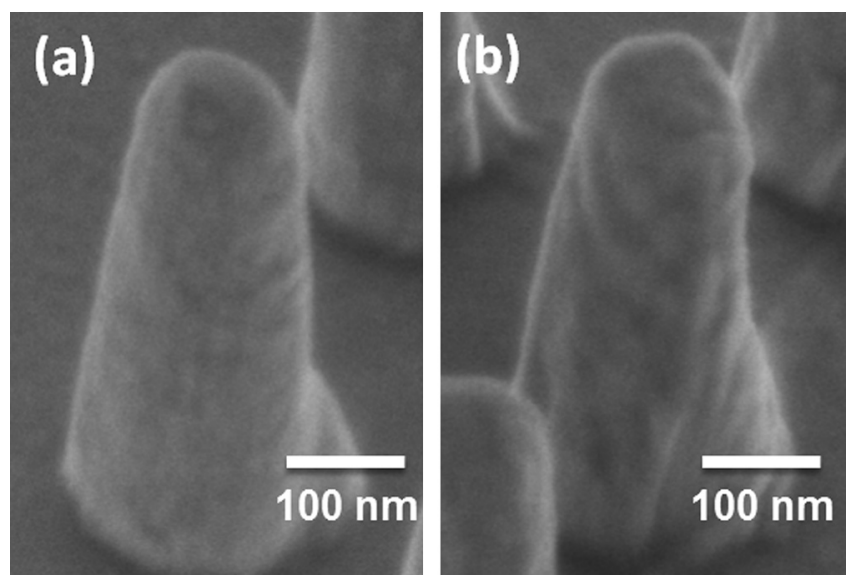

FIG. 7. Low-voltage SEM pictures showing surface profile of the nanopillar from two arrays. The two arrays are prepared with identical FIB parameters and settings, but with different $\mathrm{KOH}$ etch duration. 


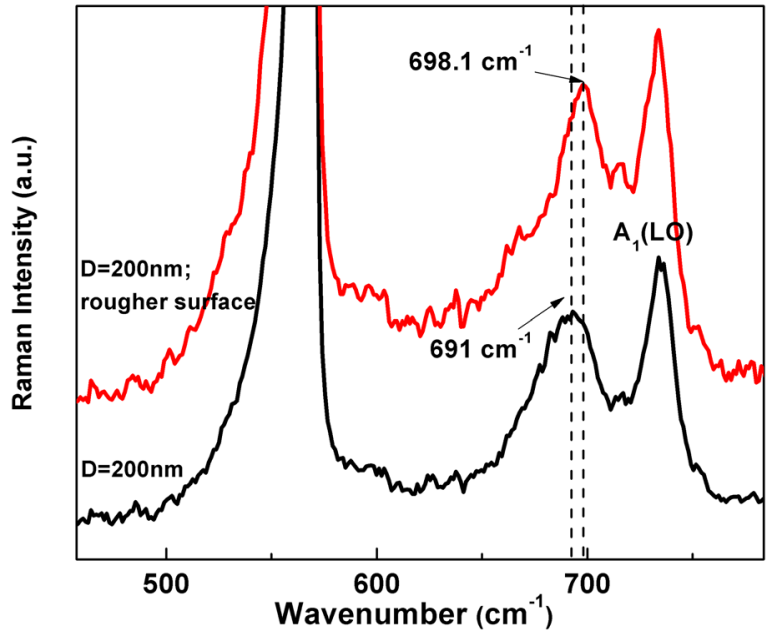

FIG. 8. (Color online) Raman spectra for nanopillar arrays with different surface roughness as shown in Fig. 7. With a smoother surface, the peak position of the SO mode downshifts $8 \mathrm{~cm}^{-1}$.

potential, hence leads to a smaller $k$ along the measuring surface. Thus the SO phonon peak in the Raman spectrum shifts further away from the $\mathrm{A}_{1}(\mathrm{LO})$ mode according to the dispersion relation.

\section{B. Temperature-variable photoluminescence}

In this section, we examine PL spectra of the nanopillar array with an average diameter of $160 \mathrm{~nm}$ at different temperatures. Comparing the PL spectra measured at $80 \mathrm{~K}$ as plotted in Fig. 9(a), the as-grown sample exhibits a single emission band centered at about $442 \mathrm{~nm}$, while two emission bands centered at about 430 and $455 \mathrm{~nm}$, respectively, are observed from the nanopillar region, which indicates a significant change of carrier localization state distribution caused by nanopillar-fabrication.

The single emission band observed from the background region is generally attributed to the emission of localized carriers confined to In-rich regions formed as a result of In component fluctuation or phase segregation within the InGaN layers. The PL spectra of the nanopillar array show the splitting of the emission spectrum into two bands. The band at the lower-energy side is broader, which is related to the confined carriers within In-rich regions, the same origin as the single band observed in as-grown background. The narrower emission band at the higher-energy side is ascribed to the emission due to another type of carriers governed by a new mechanism introduced as a result of nanostructuring. We tentatively attribute this new localization effect to boundary localization caused by nanopillar fabrication. In fact, two groups of localized state distribution were previously observed in the extremely narrow InGaN/GaN MQW structure. $^{21}$

As seen in Fig. 9(b), both the emission bands shift towards higher energy as the temperature increases, indicating the thermalization of both types of localized carriers to higher-energy states. $^{22}$ However, the band at the higherenergy side diminishes rather rapidly, becoming completely invisible at $220 \mathrm{~K}$, but the intensity of the emission at the
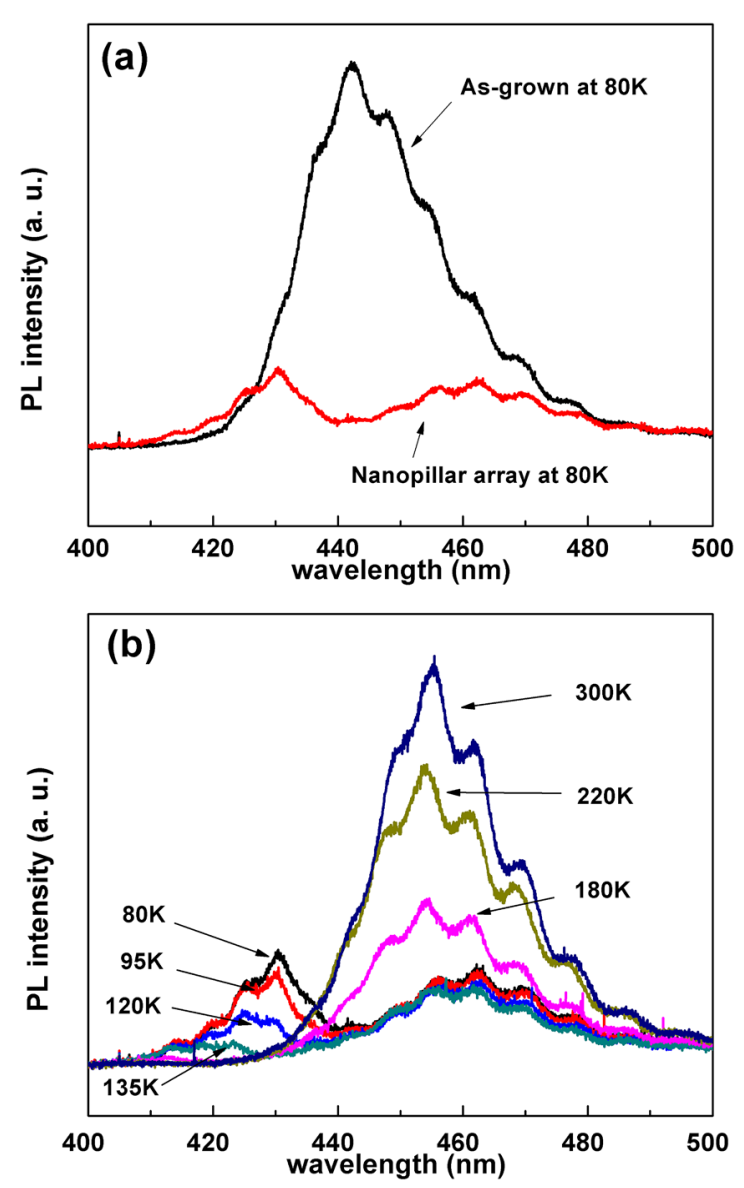

FIG. 9. (Color online) PL spectra for (a) nanopillar array and as-grown wafer at $80 \mathrm{~K}$, (b) nanopillar array with temperature varied from $80 \mathrm{~K}-300 \mathrm{~K}$.

lower-energy side increases gradually and becomes dominant at higher temperatures. The quick quenching of the PL intensity of the higher-energy band suggests its shallow localization nature. The dominant emission of the lowerenergy band at higher temperatures confirms its origin related to the localization of In-rich regions, similar to the case in non-etched regions.

\section{CONCLUSIONS}

To conclude, GaN nanopillar arrays containing InGaN/ GaN MQW have been fabricated by FIB. The ion damage by FIB is removed by subsequent wet etching. The SO phonon is observed from the Raman spectra for the nanopillars with diameters less than $220 \mathrm{~nm}$. This mode is further verified from small clusters that contain only 3-4 nanopillars, and Raman results shows that the SO mode is unrelated to the InGaN in the MQWs. The SO mode has been further investigated in nanopillar arrays with different diameter and surface roughness. Results show the SO mode shifts to lower wavenumber as the diameter decreases. For nanopillars with similar geometrical profile, the SO mode downshifts by $8 \mathrm{~cm}^{-1}$ for smoother surface. Through PL measurements, the spectrum of nanopillar array splits into two bands at $80 \mathrm{~K}$ and as the temperature rises, the high energy band quenches rapidly, indicating the shallow nature of the localized carrier. 


\section{ACKNOWLEDGMENTS}

The work described in this paper was jointly supported by a General Research Fund of the Research Grant Council of Hong Kong (Grant No. HKU 7118/09E) and the Joint Research Fund for Overseas Chinese, Hong Kong and Macau Scientists of NSFC (Grant No. 61028012).

${ }^{1}$ F. A. Ponce and D. P. Bour, Nature 386(6623), 351 (1997).

${ }^{2}$ A. J. Steckl and I. Chyr, J. Vac. Sci. Technol. B 17(2), 362 (1999).

${ }^{3}$ S.-E. Wu, C.-P. Liu, T.-H. Hsueh, H.-C. Chung, C.-C. Wang, and C.-Y. Wang, Nanotechnology 18(44), 445301 (2007).

${ }^{4}$ G. Spoldi, S. Beuer, M. Rommel, V. Yanev, A. J. Bauer, and H. Ryssel, Microelectron. Eng. 86(4-6), 548 (2009).

${ }^{5}$ Y. Yabuuchi, S. Tametou, T. Okano, S. Inazato, S. Sadayama, Y. Yamamoto, K. Iwasaki, and Y. Sugiyama, J. Electron Microsc. 53(5), 471 (2004).

${ }^{6}$ H. W. Choi, S. J. Chua, A. Raman, J. S. Pan, and A. T. S. Wee, Appl. Phys. Lett. 77(12), 1795 (2000).

${ }^{7}$ S. J. Chua, H. W. Choi, J. Zhang, and P. Li, Phys. Rev. B 64(20), 205302 (2001).

${ }^{8}$ A. Datta, S. Dhara, S. Muto, C. W. Hsu, C. T. Wu, C. H. Shen, T. Tanabe, T. Maruyama, K. H. Chen, L. C. Chen, and Y. L. Wang, Nanotechnology 16(12), 2764 (2005).

${ }^{9}$ S. Dhara, A. Datta, C. T. Wu, K. H. Chen, Y. L. Wang, S. Muto, T. Tanabe, C. H. Shen, C. W. Hsu, L. C. Chen, and T. Maruyama, Appl. Phys. Lett. 86(20) 203119 (2005)
${ }^{10}$ J. L. Weyher, S. Muller, I. Grzegory, and S. Porowski, J. Cryst. Growth 182(1-2), 17 (1997).

${ }^{11}$ M. S. Minsky, M. White, and E. L. Hu, Appl. Phys. Lett. 68(11), 1531 (1996).

${ }^{12}$ S. E. Wu, Y. W. Huang, T. H. Hsueh, and C. P. Liu, Jpn. J. Appl. Phys. 47(6), 4906 (2008).

${ }^{13}$ G. D. Mahan, R. Gupta, Q. Xiong, C. K. Adu, and P. C. Eklund, Phys. Rev. B 68(7), 073402 (2003).

${ }^{14}$ B. E. Sernelius, Surface Modes in Physics (Wiley-VCH, New York, 2001).

${ }^{15}$ M. Dutta and M. A. Stroscio, Phonons in Nanostructures (Cambridge University Press, Cambridge, 2001).

${ }^{16}$ R. Gupta, Q. Xiong, G. D. Mahan, and P. C. Eklund, Nano Lett. 3(12), 1745 (2003).

${ }^{17}$ S. Bhattacharya, A. Datta, S. Dhara, and D. Chakravorty, J. Raman Spectrosc. 42(3), 429 (2011)

${ }^{18}$ S.-E. Wu, S. Dhara, T.-H. Hsueh, Y.-F. Lai, C.-Y. Wang, and C.-P. Liu, J. Raman Spectrosc. 40(12), 2044 (2009).

${ }^{19}$ J. Q. Ning, S. J. Xu, D. P. Yu, Y. Y. Shan, and S. T. Lee, Appl. Phys. Lett. 91(10), (2007).

${ }^{20}$ T. Azuhata, T. Sota, K. Suzuki, and S. Nakamura, J. Phys.: Condens. Matter 7(10), L129 (1995).

${ }^{21}$ S. J. Xu, G. Q. Li, Y. J. Wang, Y. Zhao, G. H. Chen, D. G. Zhao, J. J. Zhu, H. Yang, D. P. Yu, and J. N. Wang, Appl. Phys. Lett. 88(8), 083123 (2006).

${ }^{22}$ Q. Li, S. J. Xu, W. C. Cheng, M. H. Xie, S. Y. Tong, C. M. Che, and H. Yang, Appl. Phys. Lett. 79(12), 1810 (2001). 